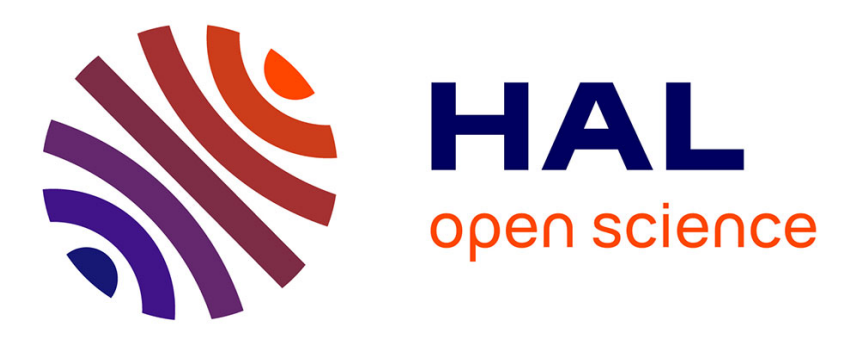

\title{
10 Design Themes for Creating 3D Printed Physical Representations of Physical Activity Data
}

Rohit Ashok Khot, Simon Stusak, Andreas Butz, Florian 'floyd' Mueller

\section{To cite this version:}

Rohit Ashok Khot, Simon Stusak, Andreas Butz, Florian 'floyd' Mueller. 10 Design Themes for Creating 3D Printed Physical Representations of Physical Activity Data. 16th IFIP Conference on Human-Computer Interaction (INTERACT), Sep 2017, Bombay, India. pp.85-105, 10.1007/978-3319-68059-0_5. hal-01679801

\section{HAL Id: hal-01679801 https://hal.inria.fr/hal-01679801}

Submitted on 10 Jan 2018

HAL is a multi-disciplinary open access archive for the deposit and dissemination of scientific research documents, whether they are published or not. The documents may come from teaching and research institutions in France or abroad, or from public or private research centers.
L'archive ouverte pluridisciplinaire HAL, est destinée au dépôt et à la diffusion de documents scientifiques de niveau recherche, publiés ou non, émanant des établissements d'enseignement et de recherche français ou étrangers, des laboratoires publics ou privés.

\section{(c)(1)}

Distributed under a Creative Commons Attribution| 4.0 International License 


\title{
10 Design Themes for Creating 3D Printed Physical Representations of Physical Activity Data
}

\author{
Rohit Ashok Khot ${ }^{1}$, Simon Stusak ${ }^{2}$, Andreas Butz ${ }^{2}$ and Florian 'Floyd' Mueller ${ }^{1}$ \\ ${ }^{1}$ Exertion Games Lab, RMIT University, Australia \\ ${ }^{2}$ University of Munich (LMU), Germany
}

\begin{abstract}
Self-monitoring technologies (such as heart rate monitors and activity trackers) that sense and collect physical activity data are becoming increasingly common and readily available. These devices typically represent the captured data using numbers and graphs that primarily appear on digital screens. More recently, representing data in a physical form such as 3D printed physical artifacts is gaining currency within HCI, owing to the engagement opportunities that come with physical representations. However, there exists a limited understanding of how to design such physical representations of personal data. To contribute to this understanding, we present a set of ten design themes, developed from the analysis of two independently designed systems that construct 3D printed physical artifacts from physical activity data. Each design theme describes a unique design feature that designers could incorporate in their design to make physical representations more engaging and playful. We envisage that our work would encourage and guide designers to think about different ways of supporting physical activity experiences.
\end{abstract}

Keywords. Physical exercise; personal informatics; quantified self; 3D printing; digital fabrication; physical visualization; self-monitoring.

\section{INTRODUCTION}

In recent years, there has been a significant surge in the sale of wearable selfmonitoring devices and smartphone-based apps that track data about an individual's active life [5,49]. Prominent amongst them are heart rate monitors [44] and pedometers [11] that enable tracking of heart rate and steps respectively. These devices predominantly use numbers, graphs and charts to represent tracked data on an accompanying smartphone screen. Individuals use this information to gain a better understanding of their active self and to utilize this gained knowledge towards making actionable changes in their lifestyle. To this end, self-monitoring is perceived as an eudaimonic pursuit [22], where the self-monitored data serves as a motivational tool for improving athletic performance $[5,33]$.

However, in line with recent studies $[5,9,10,29,47]$ we believe that self-monitored data can offer much more than just rational self-analysis. Quantification is good for 
bringing awareness and discipline to exercise, but this "number crunching" [17] activity can also make exercise feel like work. For instance, people may take more steps, or try to be more active for a while but they may not enjoy this experience for a long time [10]. This lack of engagement is evident from multiple reports that indicate abandonment of self-monitoring devices within six months of use [53]. Secondly, as Rooksby et al. [47] note, not everyone approaches self-monitoring for the sole purpose of changing one's behavior. People take interest in self-monitoring for a variety of reasons, for instance, for collecting rewards, knowing more about oneself and with an interest in exploring new technology. For such users, quantified data and its indepth analysis may offer very little value over time. Secondly, the data-centric view of representing data visually on a screen can also miss out on other opportunities of engagement that can potentially come with multisensorial representations of data, as Lupton notes [35] "numbers and graphs as a source of knowledge serve to represent bodies and selves in a very limited impoverished ways. Compare these flat forms of data materialization with the complexities of the affective embodied knowledge that is a response to a scent, taste, or the touch of skin".

Embracing the possibility of greater pleasurable interactions with one's own data, we put forward a complementary perspective on representing physical activity data through physical artifacts. We construct these physical representations using a digital fabrication process like 3D printing, that allows for an easy transformation of digital information into a physical form $[1,38]$. To this end, our perspective involves a "physical - digital - physical" mode of interaction where the physical energy of an individual is first invested to generate digital data such as heart rate. This data is then converted back into a physical form to make a re-entry into the physical world. This circular process is interesting to us because we believe closing this loop could illustrate new ways of engaging or sustaining an interest in one's data representations.

Our interest in creating physical representations also stems from the rich tactile appeal of physical artifacts $[20,23]$. For instance, unlike digital representations, the three dimensional nature of physical artifacts allows embedded data to be "touched, explored, carried and even possessed" [51]. Researchers argue that the tangible nature of physical artifacts offer possibilities to convey meaning beyond the data, which in turn might encourage people to reflect on their behavior in new and different ways, thereby yielding a more engaging experience.

Furthermore, existing literature $[24,37,52]$ also signifies the human fascination towards collecting and making artifacts. According to Miller [37], people also like to express themselves with physical artifacts that embody their lives, personalities, emotions and achievements. For example, photographs of trips and events are often printed, framed and displayed in a physical form despite the fact that they could just as well be seen on a screen. Such an arrangement of artifacts can spatially denote the identity of an individual and trigger reminiscence of good moments at a later point in time [52]. Sports in particular capitalize on such opportunities by rewarding individuals with physical trophies and medals. We believe that such physical instantiations of someone's achievements could further benefit from embedding data in them, which is now possible with the use of digital fabrication processes like 3D printing that allows creation of physical artifacts from digital designs. Consequently, design and HCI 
researchers are increasingly investigating the role of digital fabrication in HCI $[36,39,40]$, particularly towards creating meaningful manifestations of personal data $[27,30,42]$. In this regard, investigating the opportunity of digital fabrication for supporting physical activity is also timely.

Although physical representations can provide many opportunities to enrich the self-monitoring experience, there is only limited understanding of how to design such representations of physical activity data. Designers for instance, can face multiple challenges starting from how the representations should look, to what they should convey and what processes to follow in order to construct them. Prior works on ambient and physical visualizations [43,50,57] primarily focus on improving users' understanding of data through the tactile experience of handling data presented in physical form. These works however do not reveal how to apply these tactics to represent personal data generated from one's physical activity. Therefore to contribute to this understanding, we have taken a research through design [58] approach where we have independently designed and studied two systems: SweatAtoms [25] and Activity Sculptures [48] that construct 3D printed physical representations of physical activity data. SweatAtoms utilizes heart rate data of physical activity to create five physical artifacts, namely, Graph, Flower, Frog, Dice and Ring. Activity Sculptures, on the other hand, uses running data to create four physical artifacts, namely, Necklace, Robot, Lamp and Jar.

We utilized the insights gained from the respective study of SweatAtoms and Activity Sculptures, and supplemented them with the knowledge from the literature and our personal experiences in designing these systems, to develop a set of 10 design themes. Through these design themes, we unfold a rich design space [32] for creating physical representations from personal data coming from self-monitoring. Our aim is to inform designers about not only the visualization benefits of physical artifacts but also about the polyvalent values that these artifacts can offer within our everyday life. As such, we look at physical visualizations not just for visualizations but also as substances to enrich our interaction with materials and artifacts. With each theme, we articulate important design features and opportunities to create engaging physical representations. We however note that these design themes do not represent a complete set and also limited by the use of one particular technology, 3D printing to create these representations. Despite this, these themes serve as important starting pointers to inspire future investigations. Before moving to each individual theme, we describe the two systems.

\section{$2 \quad$ SweatAtoms}

SweatAtoms [23] utilizes heart rate data of physical activity for creating five 3D printed physical artifacts. Each representation reflects a different aspect of physical activity as follows (Figure 1):

Graph: The first representation is Graph, where recorded heartbeat per minute is mapped to a traditional 2D graph. We then extruded the result along $\mathrm{z}$-axis to create a 3D graph. 
Frog: The second representation is Frog, where the size of the Frog denotes the amount of physical activity done in a day. As a result, a bigger Frog means more physical activity was done in a day. We calculate the amount of physical activity based on the concept of 'active time'. An active time is the amount of time an individual spent exercising, where the heart rate was above the resting zone.

Flower: The third representation is Flower, which describes only the significant changes in the heart rate i.e. when the heart rate elevates or decreases by 20 beats per minute. We record these significant changes and map them to the length of petals, resulting in a floral patterned jewelry.

Dice: The fourth representation is Dice where six faces of the Dice describe the amount of time spent in each of the six zones of heart rate data. Each face of the Dice has a center circle, which grows in size as the user spends more time in that particular heart rate zone. By looking at the size of the central circle, one can compare time spent in each zone.

Ring: The final representation is Ring, which is a wearable ring with circles of different diameter on its periphery. The number and the diameter of each circle define the number and duration of active hours in a day. To this end, more circles mean more active hours and a circle of bigger diameter means more activity in that hour.

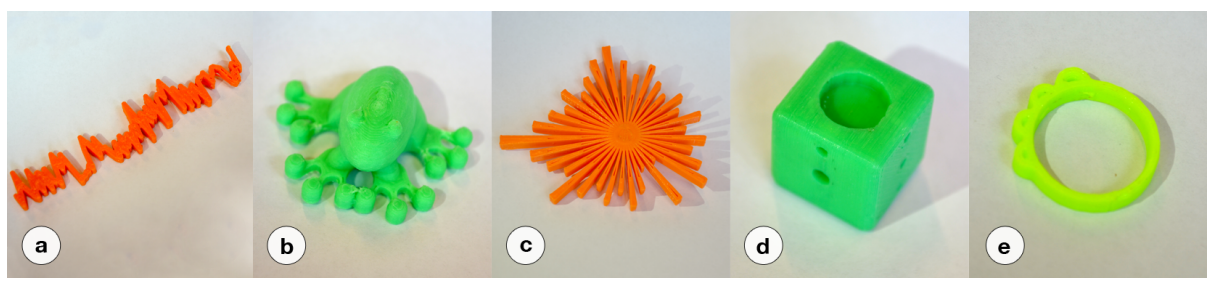

Fig. 1. SweatAtoms translates heart rate data into five 3D physical representations:

(a) Graph; (b) Frog; (c) Flower; (d) Dice; (e) Ring.

We conducted a field study of SweatAtoms system with 7 participants in 6 households to understand the impact of these representations on the behavior and experience of the participants. We installed our system along with the 3D printer in every household and asked participants to use the system every day for a period of two weeks. On each day of the study, participants wore the heart rate monitor and recorded their heart rate data for 6-7 hours during the day. In the evening, participants then $3 \mathrm{D}$ printed all five representations of their entire day's activity. To gain insights into the underlying experiences, we also asked participants to maintain a daily diary. Finally, we interviewed them in person on the first and last day of the study to gather subjective data on their experiences with the SweatAtoms system.

\section{Activity Sculptures}

Activity Sculptures [48] transforms running activity data into individual 3D printed pieces of four modular sculptures. The final four representations varied between just being decorative to having a practical purpose (see Figure 2): 
Necklace: The first representation is Necklace that contains an arbitrary number of beads, each representing one run. The size of a bead indicates the duration of the run while the shape describes distance and average running speed.

Robot: The second representation is Robot. It has eight body parts, each representing one run. Their size and shape are based on the duration, distance, calories burned as well as the average running speed.

Lamp: The third representation Lamp has ten pillars, where the difference in elevation associated with a running session is represented by the progression of a pillar. Thickness and shape are influenced by the average speed, distance, and duration.

Jar: The final representation is Jar, which is composed of an arbitrary number of round layers. The diameter of the layer is based on the duration of a running session, while the shape is influenced by the average speed and distance.

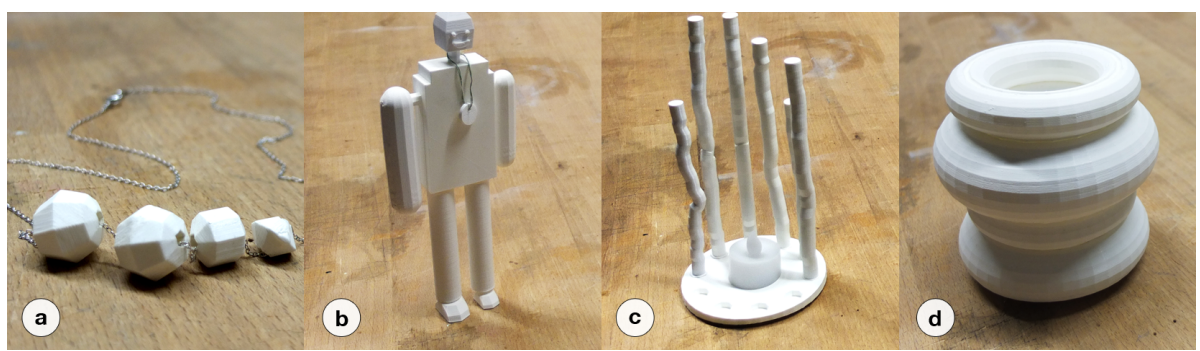

Fig. 2. Activity Sculptures are created from running data and they embed four forms:

(a) Necklace; (b) Robot; (c) Lamp; (d) Jar.

We evaluated Activity Sculptures system through a three-week field study with 14 participants. The participants used a mobile application to track their running data. In addition, participants chose the type of Activity Sculptures and the kind of delivery (personal handover or postal delivery). To gain insights on the impact of these representations, we conducted semi-structured interviews and asked the participants to fill out a questionnaire at the beginning and end of the study.

Table 1. A comparison between SweatAtoms and Activity Sculptures

\begin{tabular}{l|l|l}
\hline \multicolumn{1}{c|}{ Study Aspects } & \multicolumn{1}{c|}{ SweatAtoms } & \multicolumn{1}{c}{ Activity Sculptures } \\
\hline Physical activity data & Heart rate & Running data \\
\hline Tracking method & Heart rate monitor & Smartphone based tracking \\
\hline Tracking duration & Entire day (6-8 hours) & Exercise session \\
\hline Representations & $\begin{array}{l}\text { Graph, Frog, Flower, } \\
\text { Dice and Ring }\end{array}$ & $\begin{array}{l}\text { Necklace, Robot, Lamp and } \\
\text { Jar }\end{array}$ \\
\hline 3D printing process & Printing at home & Via manual handover or mail \\
\hline Field study & In households & In workplace \\
\hline
\end{tabular}




\begin{tabular}{l|l|l}
\hline Number of participants & 7 & 14 \\
\hline Study Duration & 2 weeks & 3 weeks \\
\hline Data collection method & Diaries and Interviews & Questionnaire and Interviews \\
\hline
\end{tabular}

\subsection{Design Process}

The foundations for this work were laid during an intensive collocated collaboration of the first two authors for three months. The core strategy that is used to build these themes was to compare and contrast findings from the two studies $[25,48]$. We used inductive thematic analysis [3] to analyse the transcribed data from both the studies. This method was considered appropriate, as designing 3D printed physical representations is still an emerging topic, for which limited relevant theory exists. The data contained transcriptions of audio recordings of 21 participants (7 from the SweatAtoms, 14 from the Activity Sculptures). First and second author independently read all transcripts a number of times to generate an initial set of codes. The codes were written on index cards along with descriptions and exemplary participants' quote. These codes were examined, cross-referenced, sorted and further analysed for overarching themes in close discussions between the first two authors. We repeatedly reviewed and refined the themes in order to maximize internal homogeneity and external heterogeneity. We combined similar themes into overarching themes and also drew coherent links or distinctions between them. These themes were further discussed and refined in a face-to-face meeting with the remaining two authors and narrowed down to a final set of 10 themes. We next invited 7 participants (4 male) from the surrounding research community to a focus group discussion, where our aim was to collect feedback on the wording, structure, and usability of the themes. Based on their feedback, we rephrased titles for some of the themes. For example, the theme 'self-expression' was initially titled as 'identity', which participants found ambiguous and they were worried that it might carry different meaning to different people. During the discussions, participants also brought up related topics such as 'shapechanging interfaces' to resolve questions raised in theme 'attachment', which we incorporated in the writing. The final set of 10 design themes is presented next.

\section{Design Themes}

We present 10 themes that describe key qualities of physical representations that designers might benefit from considering in their designs. These themes also highlight the tradeoffs and tensions in making any such decisions related to the design as identified through the field study of SweatAtoms and Activity Sculptures.

There is no predefined order in which a designer should make use of the design themes. Rather, we offer designers flexibility to start with any theme and work their way through all the themes. It is also not mandatory that the final design for the physical representation should include all the themes. We however recommend that de- 
signers should at least discuss each theme before they embark on the development stage. Designers can also utilize them to analyze their existing design choices and to identify if they satisfy the intended objectives. Table 2 describes the 10 design themes at a glance but in no particular order.

Table 2. Ten design themes for creating 3D printed physical representations

\begin{tabular}{l|l|l}
\hline$\#$ & \multicolumn{1}{|c}{ Design Themes } & \multicolumn{1}{c}{ Theme Description } \\
\hline 1 & Purpose & Intended purpose for creating physical representations. \\
\hline 2 & Data & $\begin{array}{l}\text { Aspects related to the data used to create physical rep- } \\
\text { resentations such as its type and quantity. }\end{array}$ \\
\hline 3 & Physical properties & $\begin{array}{l}\text { Physical properties used for representing data, e.g. size, } \\
\text { texture. }\end{array}$ \\
\hline 4 & $\begin{array}{l}\text { Information map- } \\
\text { ping }\end{array}$ & $\begin{array}{l}\text { Internal mapping between data and physical representa- } \\
\text { tions. }\end{array}$ \\
\hline 5 & Practical purposes & Additional utility value of physical representations. \\
\hline 6 & Self-expression & $\begin{array}{l}\text { Expressive qualities of physical representation to sup- } \\
\text { port one's identity. }\end{array}$ \\
\hline 7 & Fabrication process & Method used to create physical representation. \\
\hline 8 & Timing & Point in time when physical representations are created. \\
\hline 9 & Context & $\begin{array}{l}\text { Contextual settings that affect interactions with physical } \\
\text { representations. }\end{array}$ \\
\hline 10 & Attachment & Level of engagement with physical representations \\
\hline
\end{tabular}

\subsection{Purpose}

The first design theme focuses on the objective behind creating physical representations. For example, the objective can be to help people start or maintain a physically active lifestyle. However, this rather broad purpose can be fulfilled in various ways based on the individual user's needs [41]. For instance, users who want to change their behavior but do not commit to taking action could benefit from rewards as incentives $[33,41]$. On the other hand, users who are already physically active on a regular basis are perhaps more interested in keeping track of their progress [5,31]. To this end, we discuss four possible ways of supporting Purpose by: eliciting richer reflections on data, rewarding activity, keeping track of progress and giving testimony to invested efforts.

The purpose behind creating SweatAtoms, for example, was to elicit richer reflections on data. As a result, SweatAtoms were created from the heart rate data of an entire day's activity and not just from a one physical activity session. Secondly, each of the chosen five representations convey a different piece of information about phys- 
ical activity as understood from the gathered data. Activity Sculptures on the other hand relied more on the rewarding component where the aim was to influence and motivate running activity by rewarding each run with an individual piece of the modular physical representation. Studies of the two systems revealed that users liked and approached these representations as rewards or external push. For instance, participants of the Activity Sculptures study mentioned that the urge to receive all the pieces of the modular design served as a motivational factor for them to run more. In SweatAtoms, four participants also did more exercise to get a bigger Frog as a reward. However, intrinsically motivated participants were more interested in keeping track of their progress, hence their focus was on the information aspects of the representations. For instance, In SweatAtoms, by stacking different sized Frogs, or comparing printed Graphs from different days, participants were able to track their progress over time (see Figure 3), which they liked. Besides Frog, participants also admired the Graph and Flower as they offered insights into one's activity at a snapshot.

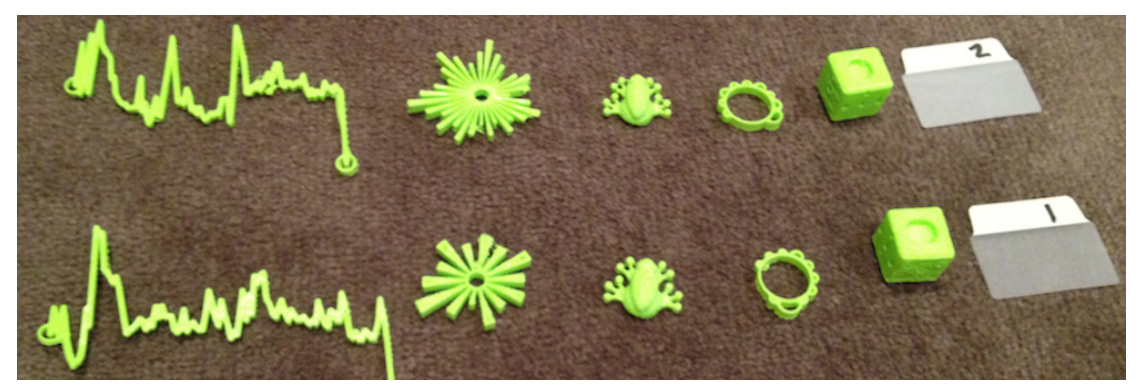

Fig. 3. Participants treasured getting physical artifacts as rewards to their efforts. Secondly by stacking them next to each other, participants were able to track their progress.

We also found that participants treasured receiving a physical reward as a testimony to their invested physical efforts. Inline with earlier study findings, physical rewards felt more satisfying than its virtual counterparts [41]. Although these physical rewards provided more of an extrinsic motivation, they in a way supplemented the pre-existing intrinsic motivations of several of the users. For five participants of SweatAtoms, physical rewards contributed to an increase in physical activity. Participants appreciated getting a bigger Frog although printing a bigger Frog also required more printing time. To this end, the perceived value of the physical representation also increased over time as participants eagerly waited for them to be printed. In future, it could be interesting to examine the relationship between printing time and size of the reward.

\subsection{Data types}

The second design theme draws designers' attention to selecting the right type of data for creating physical representations.

Let us start by looking into the data type. Physical activity typically can be measured in terms of physiological outputs (e.g., heart rate data) or movement based data 
(e.g., distance, speed). Our studies suggest that the selection between the two is important, as it could affect how individuals would perceive the constructed artifacts. For instance, SweatAtoms used physiological data in the form of heart rate to construct physical representations. In contrast, Activity Sculptures used several movement data variables such as duration, distance and average speed of a running session for creating physical representations. There are however, concerns with using heart rate data to represent one's physical activity level. Heart rate is not always a true representative of one's physical performance. An individual's heart rate might also increase in response to stress, anxiety and shock [55]. However, to obtain this level of detailed analysis would require more explicit interventions in one's life and greater logging commitments from participants. Movement based data in comparison is less ambiguous and more specific to physical activity.

The accompanying study of SweatAtoms revealed that the use of heart rate generated empathy towards the constructed physical representations as users could relate to these representations as "tokens of their heart". Participants showcased self-esteem and pride in showing them around. Activity Sculptures, which are constructed from the running data, on the other hand, did not gather a similar appeal amongst participants. Rather, these artifacts were looked at primarily for analytical purposes.

The selection of the data type can also be based on the comfort and convenience of the tracking method [2]. Physiological data requires the use of a monitoring device such as chest worn heart rate monitor, which can be awkward with a burden of carrying an additional device around. In contrast, Activity Sculptures study used the smartphones that participants already own to track the running data. Wrist worn smart watches that could now detect physiological signals could offer less awkward way of measuring physiological data.

Finally, designers should also consider ways to normalize data and prepare it into a form that is suitable for visualization [23]. In SweatAtoms, the raw heart rate data was classified into six heart rate zones, which was then used to define the shape of different representations. Similarly, in the Activity Sculptures system, the elevation gain data was normalized for 3D printing because of its large variances. Similarly, granularity of data can also be explored through the printing of interlocked physical artifacts, where each artifact can carry a different data set. Unlike screen-based systems, physical artifacts can afford distributed interactions with other artifacts in a large space [21]. Users can thus join, interlock, and mix physical artifacts to generate additional meaning [27].

\subsection{Physical properties}

This design theme looks into the physical properties of the material when designing physical representations. According to Zhao et al. [57], physical properties of a material can be split into four dimensions: 1) Geometrical dimension: e.g. volume, shape or texture 2) Physical-chemical dimension: e.g. color, weight, temperature, hardness or moisture 3) Emotional dimension: e.g. comfort, elegance 4) Associative dimension: subjective comparison to existing things of the perceiver's experience, e.g. tangible qualities such as feather-like or silky touch. 
While designing physical representations, it is important that designers pay attention to the above-mentioned dimensions to support self-expression and information mapping (discussed as subsequent themes). In SweatAtoms and Activity Sculptures, we used recyclable ABS and bio-gradable PLA as printing materials. In terms of the associative dimension, both ABS and PLA have a rigid and firm feel when touched. Participants however preferred the use of PLA to ABS as PLA is more sustainable and does not cause a striking unpleasant smell like ABS during printing. In terms of geometric dimension such as volume, both systems followed a simple mapping where the size of an artifact increased based on the amount of activity. Participants appreciated this kind of mapping. For instance, a participant from the SweatAtoms study said after receiving a Frog printed from her data, "It is like burning your body fats and putting them onto the frog." In terms of physical-chemical dimension, Activity Sculptures system used only white filament for all the representations, whereas SweatAtoms gave users the option to choose plastic filaments from a set of colors (red, green, blue, yellow and white). Participants liked the option of changing filaments as it helped them to segregate and categorize artifacts from different days.

Our choices for geometrical and physical-chemical dimensions were also restricted by the available technology. The state-of-the-art 3D printers, for example, cannot control variables such as temperature or moisture. Printing with more than one type of material at a time is also a challenge. However, with increased interest of both academic and industrial communities into digital fabrication [40], we expect that most of these challenges will be resolved in the near future. The ongoing research in shapechanging interfaces [46] and 3D printing [39] might also unveil new ways to incorporate physical properties into the design.

\subsection{Information mapping}

The fourth design theme describes the importance of internal mapping between the data and the physical representation. Finding a suitable mapping the digital data to a physical representation can be challenging, as a standard encoding process is still an area of ongoing research [23].

Drawing from our study insights, we put an emphasis on creating physical representations that are simple and easy to interpret. Common methods of representing data such as using numbers or graphs are often difficult to interpret with increase in data points as most users lack statistical skills $[7,9,13]$. Instead designers can think of using metaphors for representing data in an easy-to-interpret form. Additionally, metaphors might also offer an advantage that users might develop an empathy with them [32]. However, while choosing metaphors, designers must consider the metaphorical distance [6] between a chosen metaphor and the underlying data, as it can affect interpretive qualities of the representation.

In our systems, we used known metaphors such as flower, lamp and frog and kept the mapping simple. In the majority of the selected representations, an increase of physical activity resulted in a bigger physical artifact. However, in the Activity Sculptures project, the various data dimensions were further used to influence the shape of the physical representation, whereas in SweatAtoms, some of the representations were 
selected to emphasize and map different aspects of the heart rate and its corresponding zones. For example, Ring and Dice described the active and sedentary time based on heart rate zones.

The study results reflect that participants were happy with the choice of metaphors that offer abstract information about their activity in an aesthetically pleasing way. The fact that these representation required personal knowledge to be interpreted seemed to have fostered reflection on data. It led participants to engage with the artifacts, by observing them, testing their limits or comparing them. It also meant that participants could display them freely without feeling as if they were over-exposing themselves. For example, Frog from SweatAtoms was the favorite of all representations despite the fact that it contains very little information about physical activity (see Figure 4). It was readily displayed in people's surroundings and facilitated healthy competition among participants in order to get a bigger Frog. More informative models like Graph and Dice did not become a part of people's home ambience. This suggests to us that while mapping the data, paying attention to the aesthetics of the physical representations is important, although the embodied information can be as brief as "I did more physical activity than yesterday."

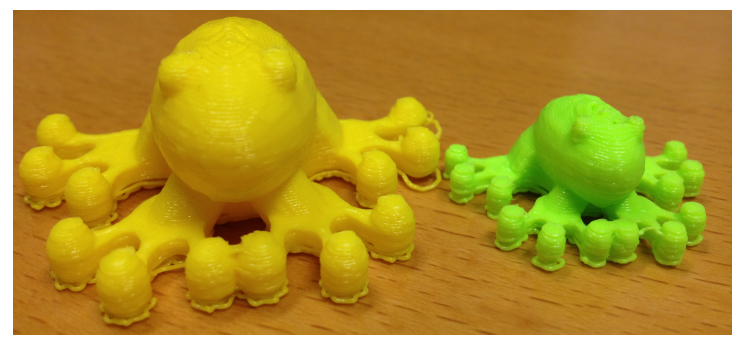

Fig. 4. Participants were happy with the choice of metaphors such as Frog that summarizes the amount of physical activity done in a day but in an aesthetically pleasing way.

Secondly, the mapping is also dependent on the quantity of the data. SweatAtoms used only one type of data, i.e., heart rate, which participants found relatively easy to map and understand. However, Activity Sculptures had a variety of data about individual's movement embedded within the artifacts. Some participants struggled to understand all bits of captured information (particularly in the beginning of the study) and how different shapes represent different pieces of information. However, these difficulties diminished as participants received more representations of a similar shape (see Figure 3).

Participants also devised their own ways of inferring and keeping track of the communicated information. In Activity Sculptures one participant wrote the number of the runs at the bottom of each piece of the Lamp figure to allow him to track the order. While another participant spelled out a strategy on how to arrange the beads of her Necklace by putting the biggest piece in the middle and the others around it. As such, the physical nature of the artifacts made it easier for participants to discover meaning through handling and playful exploration. However, embedding too much information within artifacts can make them less readable. Instead, designers could 
focus on representing a summary or only key bits of information using easy to interpret metaphors, in line with earlier study on self-monitoring devices [29]. To this end, a designer should utilise an information mapping that brings out the intended purpose of the representation. For example, a representation with the purpose to track progress should choose a mapping that makes it easy to compare different instances and encode those data variables that a user wants to improve on.

\subsection{Practical Purposes}

This theme is concerned with additional utility value of the physical representation, going beyond its main purpose. The advantage of physical representations over its digital counterparts is that they can also fulfill other practical purposes in everyday life [51]. While creating physical representations, designers could think about possible uses for these representations but users can also bring their creativity and imagination into play to devise new ways of using the representations in their life.

While designing these two systems, we had several practical purposes in mind for some of the selected representations. For example, we designed the Robot and Frog to serve as a decorative object, while we designed the Necklace, the Ring and the Flower as jewelry. The Lamp and Jar also have practical value in domestic life (see Figure 5). These prescribed uses helped participants in finding the appropriate place to keep and interact with the artifacts. For example, the Frog and Flower were placed near the desk. Necklace and Flower were worn by participants. Representations that had little practical utility, however, gathered less interest from participants, for instance Graph.

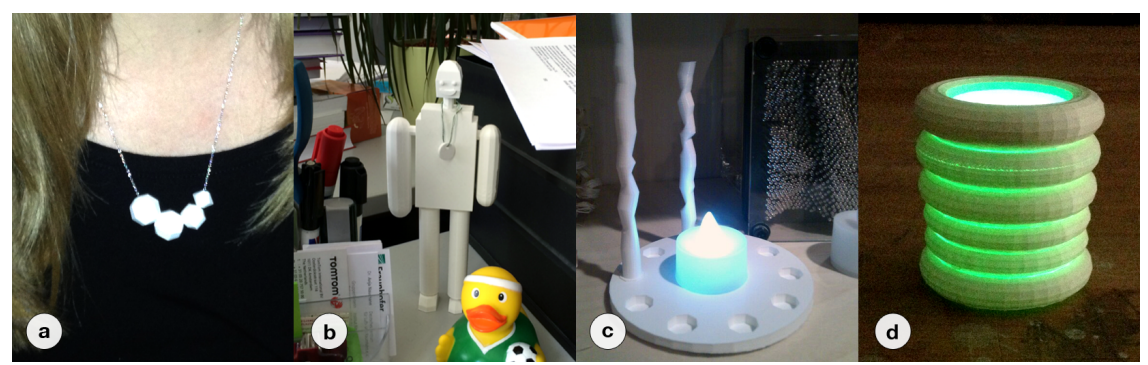

Fig. 5. Activity Sculptures were embedded in everyday life: (a) 'Necklace' worn by a participant; (b) 'Robot' placed on an office desk; (c) 'Lamp' placed on a nightstand; (d) unintended use of the 'Jar' as a Lamp.

Participants were discovering new utility values for the physical representations. For example, one of the participants from the SweatAtoms study suggested using the Flower model as a floating candleholder on water. One participant stacked his Dice models like a skyscraper. To this end, in both systems, personalization was an important aspect and participants loved to style and utilize the physical artifacts according to their needs and creative abilities.

Designers should therefore consider how users could find additional utility for the physical representations in everyday life. The user may not necessarily utilize the 
artifact in accordance with the use intended by the designer. However, having these additional uses prescribed in the design could increase user's interaction with the artifacts and thus supporting the main purpose and overall engagement with the physical representations.

\subsection{Self-Expression}

This theme looks at expressive qualities of physical representations, synonymous with the individual's understanding of his or her self $[14,35]$. Since the physical representation is an external manifestation of an individual's data, its relation with the individual can be dependent on how reflective the representation is of an individual's identity.

To support such reflection, in the SweatAtoms study, we used the fact that bodily responses to physical activity, such as heart rate values, are not only different for each individual, but also vary based on different types of physical activity. The Flower artifact as such was designed to signify these unique aspects of the individual's heart rate. Significant increases or decreases in the heart rate were mapped to an evolving floral pattern. Since these shifts in heart rate differed from person to person and also from one day to another, the resulting floral pattern in the Flower artifact was different for different days for each participant, creating a unique representation of individual's heart rate data (see Figure 6.a).

To this end, when users first encountered these artifacts, they experienced emotional responses such as intrigue, joy, disappointment and satisfaction. As Desmet [8] argued, these responses were based on not only the visceral, but also the behavioral and reflective components of individual's cognition. They appreciated them because these artifacts embodied their personal data and represented past activities, something that cannot be "bought at a shop". In Activity Sculptures, participants liked Necklace because it can be worn on the body and therefore easily be seen and shown to others. They often discussed with their families and visitors how these representations made sense in their life. The aesthetic properties of physical representations also gave users opportunities to be self-expressive, drawing from Goffman's theory [14] that any physical artifact, if put on display, becomes the public representation of the self and craftsmanship. Both systems thus highlight a design quality: uniqueness that goes beyond the traditional design strategies for digital representations. Designers should try to incorporate individual's traits in the data in order to increase appeal for these artifacts.

Most participants in both studies were vocal and enthusiast about designing representations for themselves. Participants also altered the way in which they printed and used their representation, not only to improve their understanding but also to reflect their personality. In the SweatAtoms study, some participants also chose material filaments from the given pool of material filaments to match their favorite color. Three participants wanted to change the filaments color every day so that there is a distinctive feel and order to the representation once assembled together. In the Activity Sculptures study, one participant thought about personalizing his Robot sculpture by dressing it in a shirt with a name or number while another participant from the 
SweatAtoms study created a clock with all artifacts from the study (Figure 6.b). Therefore, involving users in the design process might unveil new ways of supporting selfexpression.

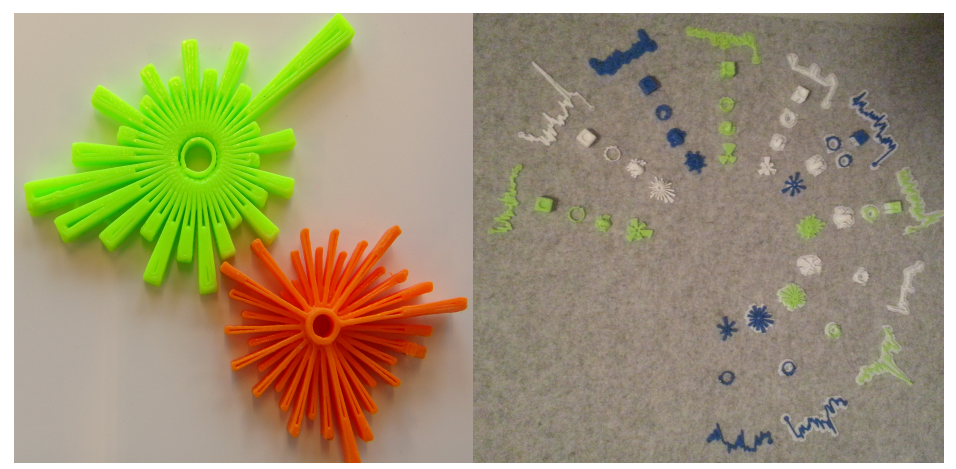

Fig. 6. a) The visible differences in Flower pattern offered insights into how heart rate changed on different days. b) Participants were also creating in using physical artifacts: one participant from the SweatAtoms study created a clock from his 3D printed artifacts.

\subsection{Fabrication process}

This theme is concerned with aspects related to the physical creation of the artifacts. Although we currently limit our discussion to one of the digital fabrication processes $-3 \mathrm{D}$ printing - the theme described here might also be relevant to other fabrication techniques such as laser cutting.

As Jansen et al. [23] have argued, the assembly and manufacturing features of physical representations can play an influential part in engaging participants with their data along with the design goals and the aesthetic features. Unlike screen based representations, creating physical representations using processes such as $3 \mathrm{D}$ printing takes time. Therefore, it becomes an important decision whether individuals should be involved in the printing process. In SweatAtoms, participants printed the artifacts in their home whereas in Activity Sculptures, we did printing at our side and then handed over the printed artifacts to the participants. Both these approaches had reasonable success. In SweatAtoms, although participants liked printing artifacts at home, their interest in the printing process faded with time, mainly because of the noise and the unpleasant smell of plastic during printing.

Despite this shortcoming, involving users in the creation process of physical representations was found advantageous as this gave users more time to anticipate and reflect on their data, thereby possibly increasing the potential value of these representations. This happened because users felt more ownership and responsibility, as they were able to see directly how their physical representations are produced from their own data [12]. Earlier research [24, 52] highlights that physical artifacts become mementos by virtue of the time and emotion invested in them by their owner. Creating an artifact can be an enjoyable experience, giving individuals the feeling of wonder, agency and satisfaction [12]. Thus, it is not usually the physical characteristic of the 
artifacts that make them biographical, but the meaning imputed to them by users as their significant personal possession.

Involving the user in the creation process however requires that the user has access to a $3 \mathrm{D}$ printer and suitable materials at home, as well as the time and necessary skills to $3 \mathrm{D}$ print these artifacts. Effort is needed on behalf of the designer to ensure that the process is user-friendly and less prone to error. Although there is rapid progress in the advancements of 3D printers, the printing time, noise and printing costs are still limiting factors, which should be kept in mind. Similarly, it is important to realize the limitations and capabilities of 3D printers and understand what kinds of physical representations are printable. Both projects, for example, used rather simple shaped and small objects for 3D printing to keep time and costs reasonable.

\subsection{Timing}

This theme talks about how often and when the physical representation should be printed or received. Physical representations can be printed during or after a physical activity.

If the 3D printing takes place 'during' the physical activity, then the user can directly influence the final shape of physical representation by changing the course of the activity. For example, a user can intentionally control the heart rate responses to influence the design of Flower. However, this would assume that the physical activity takes place near the fabrication process such as $3 \mathrm{D}$ printing, which is not always suitable as dynamic updates to $3 \mathrm{D}$ printed models in runtime is challenging and would also require technical expertise to avoid runtime printing errors. As a result this option is less viable.

Feedback "after physical activity" allows for the possibility for users to think and reflect on their physical activity performance and make different choices next time they partake same activity. Supporting this, both SweatAtoms and Activity Sculptures chose the final option and offered delayed feedback on physical activity.

In the SweatAtoms study, users printed artifacts of their data in the evening whereas in the Activity Sculptures study, participants received the artifact via mail or through a personal handover on the next day. Giving feedback in a delayed manner kept participants anticipating, unlike normal smartphone based apps where the continuous stream of data is just a glance away [15]. This process thus allowed them to concentrate more on the activities at hand and somewhat altered their habit of frequently checking their phone for feedback on the data. In SweatAtoms, participants wanted to get a bigger Frog. Since there was uncertainty in accurately predicting whether they had done enough activity to receive better looking physical representations, participants continued to remain active throughout the day and unknowingly did more exercise. Participants in the Activity Sculptures study stated that a delay between the run and the reward was beneficial, as it leaves room to speculate how the run would be reflected in the physical representation and furthermore act as an additional reward for the run. The timing also depends on the printing process itself. Participants in the SweatAtoms study had to print the physical representations manually and could choose the timing of the printing process on their own, which they liked. 
Following the work on slow technology [16], we emphasize the need for thinking about reflection and contemplation in opposition to efficiency and performance while designing physical representations. Interestingly, recovering and gaining personal health is also a slow and steady process, which demands time and consistency in the effort from the user [56]. For example, the result of burning body fat through exercise is only visible after the user has followed the exercise regularly and for a sufficient amount of time. Apparently, the 3D printing process is also quite slow at this moment. Efforts were being made to tackling the slowness of 3D printing and make the digital fabrication process more efficient [39]. In contrast, we suggest designers to embrace the slowness as a useful design resource to support a delayed feedback on physical activity data.

\subsection{Context}

This theme identifies the role of physical representations in the external schema of things, based on the context and their association with other artifacts and people. Borrowing Goffman's [14] analogy of public and private stage, there is a clear distinction in terms of how an individual presents himself to others in a public setting and in a personal private setting. A physical representation similarly has a different role to play in a public and private context. For example, placing a physical representation in a home ambience has to satisfy and cater to existing aesthetic details and surroundings, while an object that is worn on the skin should match the body types, comfort and dressing styles. It is therefore essential to customize and design such representations that can cater to a particular context of use.

The context is also crucial to understand and determine the level of abstraction required while mapping data to the representation. For example, an individual might desire more abstraction and privacy in a public setting whereas the same individual might appreciate a more detailed mapping between the data and physical representation to enable an in-depth understanding about him/herself in a private setting. Within our studies, we found that participants liked the idea of having a physical object, which cannot be identified as being easily related to their physical activity levels and therefore its significance is only revealed if the user wants to. As a result, they were happy to share it with others. For instance, one participant from the Activity Sculptures study said that she normally did not like to share her running data, e.g. by posting a run on Facebook, however she liked to show her Necklace as it is abstract form of self-expression, without revealing too much of her running data.

Verbeek [54] also brings forward the tendency of artifacts to adjust to and act according to the environment in which they were put in. Latour [28] suggests that artifacts work in a close relationship with people and other artifacts, creating networks that shape each other. Therefore, while designing physical representations designer should also identify and analyze how a physical representation would pair with other artifacts as well as the people who would interact with it.

The findings of the SweatAtoms study revealed that one of the reasons why participants appreciated the Frog and the Flower over other representations was that participants found immediate applications and places where they can place these representa- 
tions. For example, the Frog was placed on top of computer screen and the Flower was used as a coaster on the dining table. Similarly, public visibility of the artifacts in Activity Sculptures made it possible that others easily notice the data and serve as a conversation starter. These artifacts generated a sense of curiosity and sparked conversations among the visitors who did not know what the design actually meant. Participants were enthusiastic to explain the meaning to them. Some participants also gave these artifacts to their loved ones as a gift. Although, most of the physical representations from the studies worked as standalone artifacts, participants suggested to us that they would love having artifacts that can easily pair with things that they already have. In the future, it would be interesting to create artifacts that represent data from multiple people and see how it would facilitate social interactions and shared understanding of each other's data.

\subsection{Attachment}

The last theme points to the longevity of an individual's relationship with the physical representations. Previous literature on archiving suggests that physical artifacts have a longer life and value in people's life than its digital counterparts [24,52]. However, with physical artifacts there is always a burden to keep them, moreover creating and destroying them is not as easy as a digital object as argued by Kirk and Sellen [24]. As a result, designers should focus on how these representations could be able to sustain a user's interest over a longer period.

To answer this question, designers must look into the frequency of users' interactions with physical artifacts. A digital representation on a smartphone, for example, affords multiple moments of engagement at any moment in time as most users carry such devices with them and frequently interact with them on regular basis. An individual's interaction with physical artifacts is often ad-hoc, as these artifacts have a tendency to disappear in the surrounding [37]. However once noticed, these interactions with physical artifacts seem to last for a relatively longer duration. To this end, the location and the practical utility of the physical artifacts play a crucial role in determining the frequency of interaction and thus the level of one's attachment with these artifacts.

With physical representation also comes the issue of environmental sustainability, where producing plastic artifacts increase the amount of products in the environment. One of the main benefits of using digital media for representations lies in its dynamic properties: most data visualizations work supports runtime updates to visualizations whenever new data comes in. These visualizations also allow users to manipulate the view to match their interest. Physical representations are different in that they are extremely static and hard to update once they have been printed. To this end, one must print a new representation whenever new data is generated. Besides, mobile activity tracking, remote data storage and processing also consume great deals of energy.

To overcome this concern, physical representations should use biodegradable materials like PLA and allow the recycling and reuse of 3D prints. Selective printing is also an option. For example, designer can chose one type of physical representation 
for a specified goal and once user achieves this goal, another representation can be unlocked, following well-known gamification principles, e.g. unlocking new levels or badges after attaining specific achievements. Other interesting directions to resolve this issue would be to allow people to repurpose these representations, or use perishable materials like food to represent data [26]. Designers can also look into creating dynamic physical representations that augment themselves over time rather than printing new artifacts each time. For example, using shape-changing interfaces [46] that can update its shape in accordance with the changes on the personal data.

\section{$5 \quad$ Conclusion and Future Work}

This work contributes to the emerging research field of physical representations by unfolding a rich design space outlined across 10 design themes. The proposed design themes could inspire designers to think about different ways of creating representations to offer greater reflection on data. These themes not only have applications in the context of physical activity and self-monitoring, but also in other areas where personal data is of central interest e.g. recommendation systems and personalizing sports experience [27]. The presented design themes can be utilized during the ideation phase in order to come up with new design possibilities, as well as in the iteration phase where the themes could help designers in refining initial designs. Designers can use the proposed themes in any particular order and it is also not mandatory to include all the themes in one design. In order to understand how designers would make use of these themes, we are planning to conduct workshops centered on different design context such as sports and exertion games.

We also make a note that these themes were created from the design and study of two systems that construct 3D printing artifacts for physical activity data. To this end, the themes are primarily focused around digital fabrication process such as $3 \mathrm{D}$ printing to create physical representations. We invite future research towards extending these themes to other contexts and by discussing other methods (e.g., CNC milling, laser cutting) of creating physical representations. Furthermore, by including reflections from other physical representation systems, such as Patina Engraver [30], EdiPulse [26] would broaden the scope of this work.

To conclude, we invite designers and researchers - both in academia and industry alike - to challenge their notion of representing physical activity data to users on screen. As technology evolves, it is important that we as designers harness and explore the exciting opportunities that emerging new technologies like 3D printing can offer. By presenting the design and study of two systems that utilizes 3D printing, a technology, we imagined a possible design future, where individuals can create artifacts of their choice at home with their own data. To this end, we would argue that the proposed themes not only embarks the first conceptualized approach to the design, but also paves the way for future explorations in this context. 


\section{REFERENCES}

1. Anderson, C. 2010. The new industrial revolution. Wired magazine, 18, 2.

2. Benson, R. and Connolly, D. 2011 Heart rate training, Human Kinetics.

3. Braun, V. and Clarke, V. 2006, Using thematic analysis in psychology. Qualitative Research in Psychology, 3(2), 77-101.

4. Card, S., Mackinlay, J. and Schneiderman, B. 1999, Readings in information visualisation. San Francisco: Kaufmann.

5. Choe, E.K., Lee, N.B., Lee, B., Pratt, W. and Kientz, J.A. 2014, Understanding quantifiedselfers' practices in collecting and exploring personal data. In Proceedings of the SIGCHI Conference on Human Factors in Computing Systems (CHI '14). ACM, 1143-1152. DOI:http://dx.doi.org/10.1145/2556288.2557372.

6. Clevenger, T. and Edwards, R., 1988. Semantic distance as a predictor of metaphor selection. Journal of psycholinguistic research, 17(3), 211-226.

7. Consolvo, S., McDonald, D.W. and Landay, J. 2009, Theory- driven design strategies for technologies that support behavior change in everyday life. In Proceedings of the SIGCHI Conference on Human Factors in Computing Systems (CHI'09), ACM, 405-414.

8. Desmet, P. 2003. Measuring emotion: Development and application of an instrument to measure emotional responses to products. In Funology, Springer Netherlands, 111-123.

9. Elsden, C., Kirk, D.S. and Durrant, A.C. 2015, A Quantified Past: Towards Design for Remembering with Personal Informatics. Human-Computer Interaction, 31(6), 518-557.

10. Etkin, J. 2016. The hidden cost of personal quantification. Journal of Consumer Research 42: $967-984$.

11. Fitbit http://fitbit.com.

12. Gauntlett, D. 2013. Making is connecting. John Wiley \& Sons.

13. Galesic, M. and Garcia-Retamero, R. 2011, Graph literacy: A crosscultural comparison. Medical Decision Making 31, pp.444-457.

14. Goffman, E. 1959, The Presentation of Self in Everyday Life. Penguin Books.

15. Gouveia, R., Pereira, F., Karapanos, E., Munson, S.A. and Hassenzahl, M., 2016, September. Exploring the design space of glanceable feedback for physical activity trackers. In Proceedings of the 2016 ACM International Joint Conference on Pervasive and Ubiquitous Computing (UbiComp'16), ACM, 144-155.

16. Hallnas̈, L. and Redström, J 2001, Slow Technology; Designing for Reflection. Journal of Personal and Ubiquitous Computing 5, 3, Springer-Verlag, pp. 201-212.

17. Hassenzahl, M., Laschke, M. and Praest, J. 2016. On the stories activity trackers tell. In Proceedings of the 2016 ACM International Joint Conference on Pervasive and Ubiquitous Computing: Adjunct (UbiComp'16), ACM, 582-587.

18. Huang, D., Tory, M., Aseniero, B. A., Bartram, L., Bateman, S., Carpendale, S., Tang, A. and Woodbury, R. (2015). Personal visualization and personal visual analytics. IEEE Transactions on Visualization and Computer Graphics, 21(3), 420-433.

19. Hermsen, S., Frost, J., Renes, R.J. and Kerkhof, P. 2016. Using feedback through digital technology to disrupt and change habitual behavior: A critical review of current literature. Computers in Human Behavior, 57, 61-74.

20. Hogan, T. and Hornecker, E., 2016. Towards a Design Space for Multisensory Data Representation. Interacting with Computers.

21. Hornecker, E 2005. A design theme for tangible interaction: embodied facilitation. In ECSCW 2005, Springer Netherlands, 23-43. 
22. Huta, V. 2013, Pursuing eudaimonia versus hedonia: Distinctions, similarities, and relationships. In A. Waterman (Ed.), The best within us: Positive psychology perspectives on eudaimonic functioning. Washington, DC: APA Book.

23. Jansen, Y., Dragicevic, P., Isenberg, P., Alexander, J., Karnik, A., Kildal, J., Subramanian, S. and Hornbæk, K. 2015, Opportunities and Challenges for Data Physicalization. In Proceedings of the SIGCHI Conference on Human Factors in Computing Systems (CHI '15), ACM, 3227-3236.

24. Kirk, D.S. and Sellen, A. 2010, On human remains: Values and practice in the home archiving of cherished objects. ACM Transactions on Computer-Human Interaction 17, 3, 143.

25. Khot, R.A., Hjorth, L. and Mueller, F. 2014, Understanding physical activity through 3D printed material artifacts. In Proceedings of the SIGCHI Conference on Human Factors in Computing Systems (CHI'14), ACM, 3835-3844.

26. Khot, R.A., Pennings, R. and Mueller, F. 2015. EdiPulse: Supporting Physical Activity with Chocolate Printed Messages. In Proceedings of the 33rd Annual ACM Conference Extended Abstracts on Human Factors in Computing Systems (CHI EA '15), ACM, 13911396.

27. Khot, R.A., Andres, J., Lai, J., von Kaenel, J. and Mueller, F.F., 2016, June. Fantibles: Capturing Cricket Fan's Story in 3D. In Proceedings of the 2016 ACM Conference on Designing Interactive Systems (DIS'16), ACM, 883-894.

28. Latour, B. 1999. Pandora's Hope. Essays on the reality of science studies. Harvard University Press.

29. Lazar, A., Koehler, C., Tanenbaum, J. and Nguyen, D.H. 2015, Why we use and abandon smart devices. In Proceedings of the 2015 ACM International Joint Conference on Pervasive and Ubiquitous Computing (UbiComp '15), ACM, 635-646.

30. Lee, M., Cha, S. and Nam, T. 2015, Patina Engraver: Visualizing Activity Logs as Patina in Fashionable Trackers. In Proceedings of the 33rd Annual ACM Conference on Human Factors in Computing Systems (CHI'15), ACM, 1173-1182.

31. Li, I., Dey, A. and Forlizzi, J. 2011, Understanding my data, myself: supporting selfreflection with ubicomp technologies. In Proceedings of the 2011 ACM International Joint Conference on Pervasive and Ubiquitous Computing (UbiComp '11), ACM, 405-414.

32. Lin, JL, Mamykina, L, Lindtner, S, Delajoux, G and Strub, HB 2006, Fish'n'Steps: Encouraging physical activity with an interactive computer game. In Proceedings of the 2006 ACM International Joint Conference on Pervasive and Ubiquitous Computing (UbiComp '06), Springer, 261- 278.

33. Locke, E., and Latham, G. 1990. A theory of goal setting and task performance, Prentice Hall, Englewood Cliff, NJ USA.

34. Löwgren, J. 2013, Annotated portfolios and other forms of intermediate-level knowledge. Interactions 20(1): 30- 34 .

35. Lupton, D. 2016, The Quantified Self: A Sociology of Self-Tracking, Polity.

36. Mellis, D., Follmer, S., Hartmann, B., Buechley, L. and Gross, M.D. 2013, FAB at CHI: digital fabrication tools, design, and community. In Proceedings of the 31st Annual ACM Conference Extended Abstracts on Human Factors in Computing Systems (CHI EA '13), ACM, 3307-3310.

37. Miller, D. 2010, Stuff. Polity Press, Cambridge.

38. Mota, C. 2011, The rise of personal fabrication. In Proc. Creativity and cognition (CandC '11), ACM, 279- 288.

39. Mueller, S., Mohr, T., Guenther, K., Frohnhofen, J. and Baudisch, P. 2014, faBrickation: fast 3D printing of functional objects by integrating construction kit building blocks. Pro- 
ceedings of the SIGCHI Conference on Human Factors in Computing Systems (CHI'14), ACM, 3827-3834.

40. Mueller, S., Devendorf, L., Coros, S., Ochiai, Y., Gannon, M. and Baudisch, P., 2016, May. CrossFAB: Bridging the Gap between Personal Fabrication Research in HCI, Computer Graphics, Robotics, Art, Architecture, and Material Science. In Proceedings of the 2016 CHI Conference Extended Abstracts on Human Factors in Computing Systems, ACM, 3431-3437.

41. Munson, S.A. and Consolvo, S. 2012, Exploring Goal-setting, Rewards, Self-monitoring, and Sharing to Motivate Physical Activity, Pervasive Health 2012, 25-32.

42. Nissen, B. and Bowers, J. 2015, Data-Things: Digital Fabrication Situated within Participatory Data Translation Activities. In Proceedings of the SIGCHI Conference on Human Factors in Computing Systems (CHI'15), ACM, 2467-2476.

43. Pousman, Z., \& Stasko, J. 2006, A taxonomy of ambient information systems: four patterns of design. In Proceedings of the working conference on Advanced visual interfaces (AVI'06), ACM, 67-74.

44. Polar Heart rate monitors, http://www.polar.com/products/H7_heart_rate_sensor.

45. Prochaska, J. and Velicer, W. 1997. The transtheoretical model of health behavior change. American journal of health promotion, 12(1): 38-48.

46. Rasmussen, M.K., Pedersen, E.W., Petersen, M.G. and Hornbæk, K. 2012, Shapechanging interfaces: a review of the design space and open research questions. In Proceedings of the SIGCHI Conference on Human Factors in Computing Systems (CHI'12), ACM Press.

47. Rooksby, J., Rost, M., Morrison, A. and Chalmers, M. 2014, Personal tracking as lived informatics. In Proceedings of the SIGCHI Conference on Human Factors in Computing Systems (CHI'14), ACM, pp.1163-1172.

48. Stusak, S., Tabard, A., Sauka, F., Khot, R. and Butz, A. 2014, Activity Sculptures: exploring the impact of physical visualizations on running activity. TVCG 20, 12 (2014), 22012210.

49. Swan, M. 2012. Sensor mania! The internet of things, wearable computing, objective metrics, and the Quantified Self 2.0. J. Sens. Actuator Netw. 2012, 1, 217-253.

50. Tomitsch, M., Kappel, K., Lehner, A., \& Grechenig, T. 2007, Towards a Taxonomy for Ambient Information Systems. In Ambient Information Systems.

51. Vande Moere, A. 2008, Beyond the tyranny of the pixel: Exploring the physicality of information visualization. In Proc. IV'08.

52. Van den Hoven, E. 2004, Graspable Cues for Everyday Recollecting. PhD thesis, Technische Universiteit Eindhoven, The Netherlands.

53. Velayanikal, M. 2014. Can a fitness tracker, which rewards users solve the problem of wearable tech? https:/www.techinasia.com/mymo-fitness-tracker-gives-rewards-to-users/. (2014). Accessed: 17-07-2015.

54. Verbeek, P.P. 2005, What things do-philosophical reflections on technology, agency and design. The Pennsylvania State Press.

55. Vermeulen, J., Lindsay, M., Johannes, S., Russell, B. and Sheelagh, C. 2016, Heartefacts: Augmenting Mobile Video Sharing Using Wrist-Worn Heart Rate Sensors. In Proceedings of the 2016 ACM Conference on Designing Interactive Systems (DIS'16), ACM, 712-723.

56. Weinberg, R.S. and Gould, D. 2006, Foundations of Sport and Exercise Psychology. Human Kinetics, Champaign, IL.

57. Zhao, J., \& Vande Moere, A, 2008, Embodiment in data sculpture: a model of the physical visualization of information. In Proceedings of the 3rd international conference on Digital Interactive Media in Entertainment and Arts, ACM, 343-350. 
58. Zimmerman, J., Forlizzi, J. and Evenson, S. 2007, Research through design as a methods for interaction design research in HCI, In Proceedings of the SIGCHI Conference on Human Factors in Computing Systems (CHI'07), ACM, 493-502. 